\title{
Actividad del fuego en áreas forestales de México a partir de sensores remotos y su sensibilidad a la sequía
}

\section{Remote-sensed forest fire activity across Mexico and its sensitivity to drought}

\author{
Darío Cisneros-González', José Manuel Zúñiga-Vásquezl y Marín Pompa-García|*
} l Universidad Juárez del Estado de Durango. Facultad * Autor de correspondencia. mpgarcia@ujed.mx
de Ciencias Forestales. Durango, Durango, México.

\section{RESUMEN}

Los incendios forestales en México son reguladores naturales en la dinámica de los ecosistemas forestales. Sin embargo, su mala gestión puede causar consecuencias catastróficas, que afectan a los elementos de los ecosistemas donde se presenten. Fuentes oficiales indican un incremento en los últimos años en su ocurrencia y en la superficie afectada. Estudios recientes indican que los incendios forestales están estrechamente vinculados a fenómenos climáticos. Por lo tanto, el objetivo principal de este trabajo fue evaluar la relación entre la sequía y la ocurrencia de incendios en México durante el período 2005-2016. Los datos de fuego se obtuvieron del espectrorradiómetro de imágenes de resolución moderada (Modis, por sus siglas en inglés) mientras que los datos de sequía se fundamentaron en el índice estandarizado de precipitación y evapotranspiración (SPEI). A través del estadístico G de Getis-Ord se determinó geoespacialmente donde se ubica la mayor incidencia de incendios forestales y la mayor área afectada. Se observó que los incendios se agrupan en el centro del país a lo largo de la Sierra Madre del Sur y en el Eje Neovolcánico. Además, se concluye que la sequía se ve relacionada con la ocurrencia de incendios forestales y su superficie afectada en 32\% y $38 \%$ respectivamente. Este estudio contribuye a investigaciones recientes que relacionan la influencia de los fenómenos climáticos con el fuego.

PALABRAS ClAVE: análisis geoespacial, estadístico G de Getis-Ord; Modis; recurrencia de fuego, SPEI, superficie incendiada.

\section{ABSTRACT}

Forest fires in México are natural regulators in the dynamics of forest ecosystems. However, their mismanagement can cause catastrophic consequences, affecting critical elements of the ecosystems where they occur. In recent years, official sources have reported an increase in forest fires and affected area. It is very well known that forest fires are widely linked to climatic phenomena. Therefore, the main objective of this study is to evaluate the relationship between drought and fire occurrence in Mexico during the period 2005-2016. The fire data were obtained from Moderate Resolution Imaging Spectroradiometer (Modis) while the source of drought data is from a Standardized Index of Precipitation and Evapotranspiration (SPEI). Through the G statistic of Getis-Ord it was geospatially determined where the highest incidence of fires occur and where the largest affected area is located. Results indicated that fires are clustered in the center of the country along the Sierra Madre del Sur and the Eje Neovolcánico. It is also concluded that the drought is related to the occurrence of forest fires and their affected surface by $32 \%$ and $38 \%$ respectively. This study contributes to recent research linking the influence of climate phenomena with fire.

KEYWORDS: geospatial analysis, G-statistics, Modis. fire recurrence, SPEI, burned surface. 


\section{INTRODUCCIÓN}

El estudio de los incendios forestales se considera de gran importancia debido a las pérdidas naturales, materiales y humanas que producen (Gómez-Pazo y Salas, 2017). Estos fenómenos pueden tener efectos duraderos en los ecosistemas, e incluso ocasionar impactos irreversibles en función a su intensidad y severidad (Burel y Baudry, 2002). Los incendios pueden resultar benéficos para los ecosistemas que evolucionaron con su ocurrencia (Flores, Rodríguez, Estrada y Sánchez, 2006; Jiménez y Alanís, 2011; Márquez-Linares, Jurado y López-González, 2005), pero no aquellos que no están adaptados a este fenómeno (Rodríguez-Trejo, 2001). El problema surge cuando la ocurrencia del fuego supera a las especies en su capacidad de tolerancia y se ven afectados sus procesos ecológicos (Del Campo y Bernal, 2010), lo que ocasiona pérdida de biodiversidad, deforestación, modificación del proceso hidrológico y liberación de gases de efecto invernadero (Páramo, 2007; Pérez-Verdín, Márquez-Linares, CortésOrtíz y Salmerón-Macías, 2013).

A escala mundial, los incendios forestales representan uno de los principales agentes de pérdida de cubierta vegetal (Bodí, Cerdà, Mataix-Solera y Doerr, 2012). Por ejemplo, en México en el año 2016 se registró un incremento de 56\% en la ocurrencia de incendios forestales con relación al año 2005 , así como $68 \%$ en la superficie afectada, con un número promedio de 7985 de incendios por año y 317441 ha afectadas (Comisión Nacional Forestal [Conafor], 2017). Ante estas cifras, es relevante conocer tanto la frecuencia como la superficie afectada por los incendios forestales, puesto que son dos parámetros importantes para determinar regímenes del fuego (Rodríguez-Trejo y Myers, 2010), ya que puede haber zonas con alto número de incendios, pero con poca superficie afectada y viceversa (Pérez-Verdín et al., 2013).

Si bien la presencia de incendios forestales se atribuye principalmente a factores antropogénicos (Espíndola, Trejo, Morales y Pérez, 2017), se sabe que las sequías prologadas, pueden ser también un agente causal importante para la ocurrencia del fuego (Estrada y Ángeles, 2007; Williams et al., 2015; Pérez-Verdín et al., 2013; Yocom et al., 2017; Pompa-García, Camarero, Rodríguez-Trejo y Vega-Nieva, 2017) y su impacto puede generar incendios difíciles de combatir debido a su intensidad (PeñaFernández y Valenzuela-Palma, 2008).

\section{OBJETIVOS}

Por lo anterior, se plantea la hipótesis de que existe una relación directa entre los eventos de sequía con la ocurrencia y extensión de los incendios forestales; por lo que el principal objetivo de este trabajo fue evaluar la relación de la sequía con la superficie afectada y frecuencia de incendios forestales en México durante el periodo 20052016.

\section{MATERIALES Y MÉTODOS}

\section{Área de estudio}

México se ubica en la región meridional de América del Norte, colinda al norte con Estados Unidos de América y al sur con Belice y Guatemala (Fig. 1). Cuenta con una superficie aproximada de 196.4 millones de hectáreas (Comisión Nacional para el Conocimiento y Uso de la Biodiversidad [Conabio], 2017) de las cuales 33.5 millones corresponden a bosques, 32.11 millones a selvas y 12.38 millones de hectáreas a pastizales, además de poseer 15 diferentes zonas fisiográficas (Instituto Nacional de Estadística Geografía e Informática [Inegi], 2017).

\section{Datos}

Los datos de áreas incendiadas e incendios activos a escala mundial están disponibles en línea través del sitio web de la National Aeronautics and Space Administration (NASA) por medio de datos del sensor Modis y el infrarrojo Visible Infrared Suite (Viirs) ver NASA (s/f). Para los datos de incendios activos, el sensor Modis identifica las radiaciones infrarrojas emitidas por el fuego a través de los satélites Aqua y Terra; lo cual ayuda a identificar la presencia de un incendio activo. También Modis muestra la presencia de incendios de día y noche, su ubicación y otros atributos que describen los pixeles del fuego y distingue "entre fuego", "sin fuego" y "sin observación". 

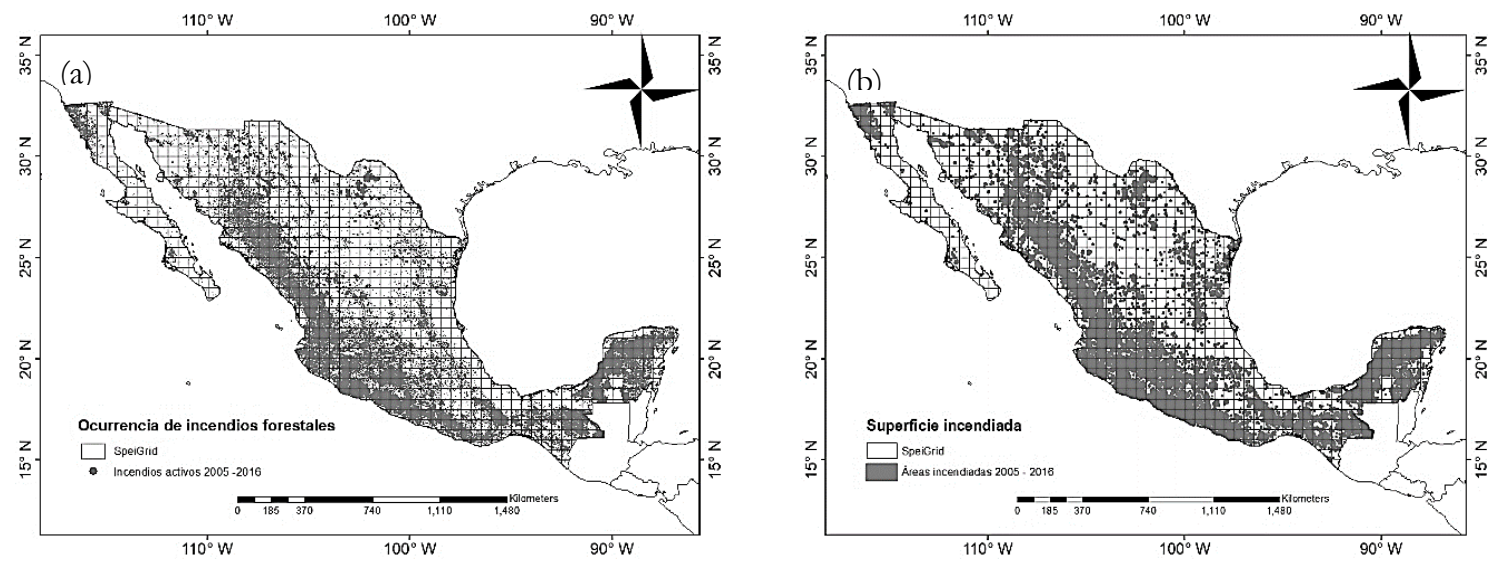

FIGURA 1. Área de estudio: (a) ocurrencia de incendios forestales 2005-2016, (b) superficie incendiada 2005-2016 (National Aeronautics and Space Administration [NASA], 2017).

Los datos de áreas quemadas se encuentran en una cuadrícula sinusoidal denominada técnicamente "grid" conformada por mosaicos de $10^{\circ} \times 10^{\circ}$, y son obtenidos con base en la información recolectada por los satélites anteriormente mencionados, sin embargo, estos datos utilizan un algoritmo que permite analizar la reflectancia superficial a $500 \mathrm{~m}$ en su dinámica diaria, lo que posibilita identificar cambios rápidos en ella y considerar el cambio de reflectancia por temperatura como la extensión de un área incendiada (Roy, Jin, Lewis y Justice, 2005).

Los datos de sequía fueron obtenidos a través de SPEI (Standardized Precipitation-Evapotranspiration Index) ver (Beguería, Latorre, Reig, y Vicente-Serrano, 2017). Este índice se define como un monitor mundial de sequía multiescalar, basado en datos climáticos que presenta pixeles con una resolución espacial de 0.5 grados y escalas de tiempo entre 1 y 48 meses, que estiman la gravedad de la sequía en función a su intensidad, duración, inicio y final de los episodios donde valores negativos indican ausencia de precipitación y valores positivos indican lo contrario (Vicente-Serrano, Beguería y López-Moreno, 2010). Para este estudio, se tomó en cuenta una escala de tiempo de sequía acumulada de 3 meses hasta el mes de mayo debido al comportamiento estacional de los incendios forestales en México (Cerano-Paredes et al., 2015), su vinculación con fenómenos climáticos (Williams et al., 2015) y patrones circulatorios a gran escala (Pompa-García y Sensibaugh, 2014). Sin embargo, queda pendiente extender el análisis en función de la variación espacio temporal de la ocurrencia de incendios como lo indica Pompa-García et al. (2017), donde se evidencia la variabilidad y extensión de la época de incendios.

\section{Geoprocesamiento y análisis espacial}

Por medio de geoprocesamientos se obtuvieron polígonos de áreas quemadas, los cuales se sometieron a un proceso de depuración a través de un mapa de uso de suelo y vegetación (Serie V) (Inegi, 2013). Se filtraron únicamente los valores correspondientes a cobertura forestal para cada uno de los años, descartándose superficies incendiadas o incendios activos correspondientes a zonas agrícolas, aserraderos, incendios urbanos y otros. Este mismo procedimiento de filtrado se aplicó también, a los datos de incendios activos para el mismo periodo de análisis.

Se realizó un análisis de puntos calientes técnicamente conocido como análisis de "hot spots" para cada año (20052016) con base en el estadístico G de Getis-Ord (Ord y Getis, 1992), el cual permite identificar tendencias de agrupamiento de valores altos o bajos, en la recurrencia de incendios forestales o áreas afectadas. La distribución dada 
por este estadístico puede ser aleatoria, uniforme o agregada y está definido por (1):

$$
G=\frac{\sum_{i=1}^{N} \sum_{j=1}^{N} W_{i j}(d) X_{i} X_{j}}{\sum_{i=1}^{N} \sum_{j=1}^{N} X_{i} X_{j}} i \neq j
$$

Donde:

$G=\quad$ Estadístico $G$ de Getis-Ord

$x_{i j}=$ medición del atributo para la unidad $i$ y $j$ respectivamente.

$W_{i j}(d)=$ medida de uno/cero en una matriz espacial simétrica para detectar la proximidad entre $i$ y $j$ y la distancia dada por $d$.

Con la finalidad de indicar que el valor determinado por el estadístico $G$ es diferente significativamente al valor esperado y diferente a la distribución al azar, se calculó el valor de la significancia estadística de la agrupación $G(Z)$, $\operatorname{con}(2)$.

$$
Z=\frac{G(d)-G_{E}(d)}{\operatorname{DesvStd}[G(d)]}
$$

Donde:

$Z=\quad$ valor de la significancia estadística de la agrupación $G$.

$G(d)=$ valor aleatorio de la prueba del estadístico $G$.

$G(d)=$ valor esperado de la prueba del estadístico $G$.

DesvStd =desviación estándar de $G(d)$.

Los valores obtenidos con este análisis (Gi Z Score) mayores a cero, revelan la existencia de agrupamiento de la variable buscada, mientras que valores cercanos a cero, indican distribución aleatoria de los valores.

El análisis se realizó con el software ArcGIS 10.0, dividiendo la República Mexicana en 852 unidades o cuadrículas con base en el grid SPEI $\left(0.5^{\circ} \times 0.5^{\circ}\right)$. Suponiendo que la presencia de incendios forestales y superficie afectada están vinculados con fenómenos climáticos, se realizó una unión espacial entre el grid del SPEI y los datos de actividad del fuego (frecuencia y extensión). De esta forma se contó el número de incendios activos y la superficie afectada por unidad o cuadrícula (en hectáreas). Se utilizaron datos vectoriales de las regiones fisiográficas de México (Inegi, 2017) para presentar los resultados por zona.

\section{Análisis anual de la relación fuego/sequía}

Una vez identificadas las zonas de agrupación de incendios forestales anuales, se obtuvo el valor de sequía acumulada de tres meses para el mes de mayo (SPEI 3) correspondiente a cada zona de agrupación. Se realizaron correlaciones de Pearson ( $\mathrm{p} \leq 0.05)$ entre los valores obtenidos de SPEI, las unidades de agrupación de incendios y la agrupación de superficies incendiadas por año.

\section{Análisis temporal de la relación fuego/sequía}

Adicionalmente se realizó un análisis temporal entre las áreas incendiadas, la ocurrencia de incendios y la sequía a través de correlaciones de Pearson. En contraste con el análisis anual, en este apartado se consideraron valores de SPEI en una escala de 1 mes a 48 meses de enero a diciembre para cada año de 2005 a 2016 sin considerar las zonas fisiográficas. Estos valores fueron relacionados con el número de incendios y las superficies incendiadas de cada mes para encontrar los meses en los que más se ha relacionado la sequía con el fuego.

\section{Resultados}

\section{Superficie incendiada}

Se encontraron zonas de agrupación de incendios y superficie incendiada en cada año analizado. La zona fisiográfica que muestra la mayor cantidad de áreas incendiadas por año es la Sierra Madre del Sur, donde los incendios fueron constantes en todos los años del análisis. Por otra parte, la Península de Yucatán, la Sierra Madre Oriental y la Cordillera Central de América del Norte son las zonas que presentan menor superficie de afectación presentándose cada una en solo uno de los años que comprende el periodo de estudio (Material suplementario, ilustración 1). 
El mayor número de áreas afectadas por zona y año se presentó en 2005 en la Sierra Madre del Sur, con 46\% más de superficie afectada en relación con 2012 para la misma zona, resultado más cercano en tiempo a 2005. Por otra parte, la Llanura Costera sur del Golfo de México mostró el resultado con menor número de áreas afectadas en 2007.

En cuanto al índice de sequía, los años con mayor ocurrencia en su valor más bajo (-2.33), fueron 2011 con dicho valor en dos de sus zonas y 2012 que lo presenta en tres de ellas. El valor más húmedo encontrado en los pixeles analizados (2.33) fue para el año 2015 en el Eje Neovolcánico Transversal. Este fue el segundo año con menos agrupación de superficies incendiadas reportadas (2 495803 ha), solo después del 2010 que mostró una agrupación en la Sierra Madre del Sur con 156125 ha.

\section{Ocurrencia de incendios forestales}

Se encontraron zonas de agrupamiento distintas al análisis de áreas incendiadas como las Sierras de Chiapas y Guatemala y la Península de Baja California. La zona más recurrente fue la Península de Yucatán, ya que permanece constante en todos los años correspondientes al análisis, seguida de la Sierra Madre del Sur, la cual no aparece en solo uno de los años del periodo de estudio. La zona menos recurrente resultó ser la Llanura Costera del Golfo Sur la cual solo aparece en uno de los mapas anuales obtenidos al igual que la Península de Baja California (Material suplementario, ilustración 2).

El mayor número de incendios se presentó en 2011 en la Sierra Madre Occidental, lo cual corresponde con lo expuesto en el análisis anterior. Sin embargo, en zonas de la Sierra Madre del Sur en el 2005 y en la Península de Yucatán en 2009, también se presentaron cantidades importantes de incendios forestales.

En 2005, la Llanura Costera del Golfo se presenta también como una de las zonas con menor número de incendios; el Eje Neovolcánico, aunque se presentó en cinco de los 12 años de estudio, generalmente mostró valores bajos, y particularmente en el 2006 se registró en esa zona la menor presencia de incendios activos.

\section{Asociación anual de sequía-superficie incendiada e incendios forestales}

Las correlaciones de Pearson indican una relación negativa entre los valores del SPEI y la superficie incendiada y SPEI con número de incendios. Se encontraron valores de $\mathrm{r}=$ 0.38 para la relación SPEI-áreas incendiadas, $\mathrm{y}$ un $\mathrm{r}=-0.32$ para la relación SPEI-fuego activo, considerando en ambos casos un valor de $\mathrm{p} \leq 0.05$ (Fig. 2).

\section{Asociación temporal de sequía-superficie incendiada y frecuencia de incendios}

Se encontraron correlaciones negativas (estadísticamente significativas) entre la sequía acumulada y el fuego. Dichas correlaciones mostraron que la sequía acumulada de tres meses, principalmente la del mes de junio (color rojo intenso, $r=-0.80$ ) ha influido en la superficie incendiada durante el periodo 2005-2016 como se muestra en la figura 3a. Respecto a la ocurrencia de incendios se encontró que la sequía acumulada de tres meses de junio y julio (rojo intenso, $r=-0.80)$ influye de manera sustancial en dicha ocurrencia.

\section{DISCUSIÓN}

El estadístico G se muestra eficiente para el análisis de la tendencia espacial de los incendios forestales (Reddy et al., 2016). Con base en dicho análisis, se encontraron zonas de agrupación para las dos variables analizadas (ocurrencia de incendios y superficie afectada), lo que demuestra que ambos sucesos se presentan no aleatorios. Sin embargo, los resultados mostraron discrepancias entre la ubicación de las agrupaciones de áreas incendiadas y la frecuencia de incendios, lo que justifica la importancia de su análisis por separado. Con estos resultados se confirma la importancia de conocer cada una de estas variables para establecer regímenes de fuego para la descripción de tendencias espaciales y temporales de los incendios forestales cuyo principal origen son causas antropogénicas (ZúñigaVásquez, Cisneros-González, Pompa-García, RodríguezTrejo y Pérez-Verdín, 2017a). Las discrepancias mencionadas pueden deberse a la carga de combustibles en ciertas zonas (Keeley, 2009), condiciones topográficas y a la variabilidad interanual del clima. 

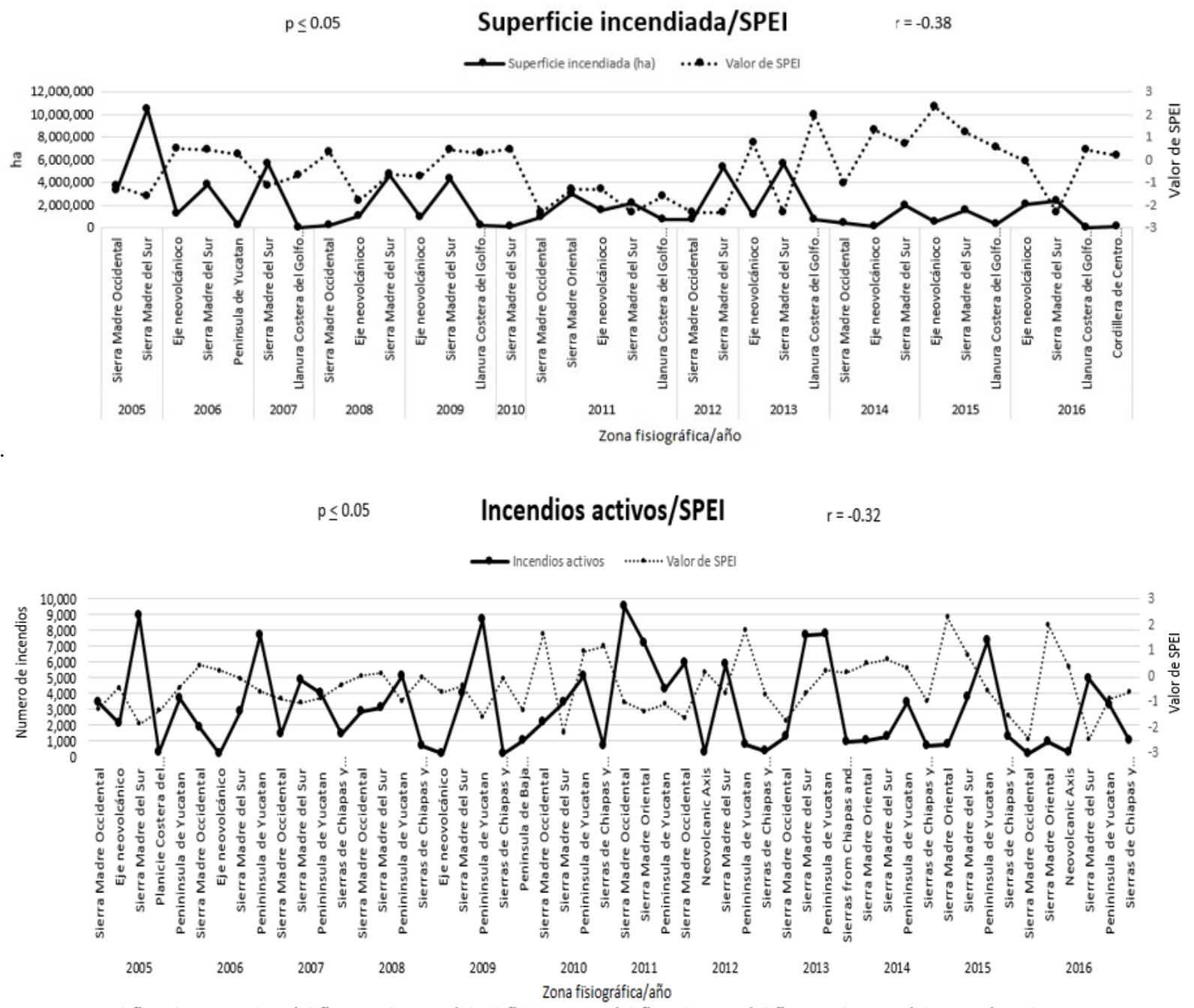

FIGURA 2. Correlación de Pearson entre sequía (SPEI) y áreas incendiadas (arriba) y fuego activo (abajo).

Los incendios forestales se ven influenciados por actividades y comportamientos humanos (Vélez, 2009) debido al cambio de uso de suelos y el abandono rural (Pérez-Cabello, Ibarra, Echeverría y de la Riva, 2010; Carreiras et al., 2014). Sin embargo, los resultados en este estudio señalan que existe una relación de estos fenómenos con la sequía, principalmente con las áreas incendiadas, fundamento destacable si se considera que $90 \%$ de los incendios son provocados por el hombre (Conafor, 2017). En otras partes del mundo diversos autores han explorado esta vinculación (Williams et al., 2015, Littell, Peterson, Ryley, Liu y Luce, 2016) y han encontrado también relaciones significativas entre la sequía y el fuego. En particular para México, el presente estudio contribuye a los estudios recientes que explican la relación de la sequía con la frecuencia de incendios forestales y las áreas afectadas por los mismos (i.e. Pompa-García et al. 2017; ZúñigaVásquez, Cisneros-González y Pompa-García, 2017b). Esto cobra mayor relevancia ante las condiciones climáticas futuras pronosticadas para nuestro país (Seager et al., 2009). Como se anticipaba, los años que presentaron mayor número de zonas fisiográficas afectadas fueron el 2011 y 2012 debido a las condiciones intensas de sequía presentadas en dichos años. De acuerdo con Castillo- 
a)
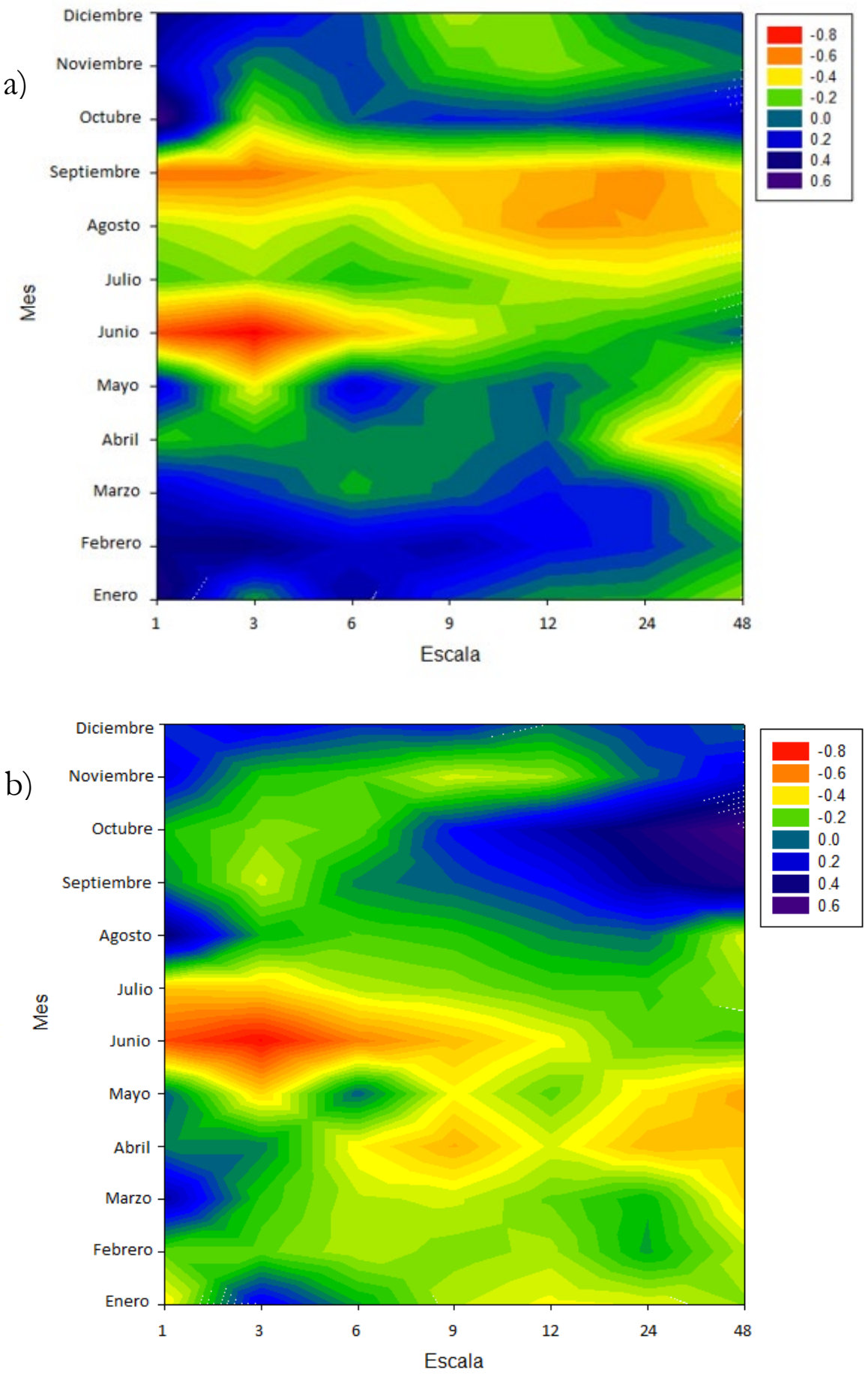

FIGURA 3. Gráfica de correlación: a) SPEI/áreas incendiadas, b) SPEI/número de incendios. Los valores de SPEI fueron obtenidos en una escala de 1- 48 meses (eje X) de enero a diciembre (eje Y) de cada año. Las gráficas muestran coeficientes de correlacion de pearson (r) usando escalas de color. Los valores son significativos $(\mathrm{p}<0.05)$. 
Castillo, Ibáñez-Castillo, Valdés, Arteaga-Ramírez y Vázquez-Peña (2017) fueron los años donde se presentaron una de las sequías más intensas, solo después de 1998 donde el año previo se produjo gran cantidad de biomasa (i.e. combustible) debido a las condiciones climáticas que prevalecieron y que mostraron su variabilidad diferenciada por la región geográfica del país.

La recurrencia del fuego puede causar daños al suelo, a la vegetación y a otros factores de los ecosistemas, lo que ocasiona un retraso en la recuperación de las áreas afectadas (Polychronaki, Gitas y Minchella, 2014), aunque cabe mencionar que esto responde proporcionalmente a la carga de combustible (Keeley, 2009). No obstante, estudios recientes señalan que la baja precipitación está ampliamente relacionada con la ocurrencia de los incendios forestales (Littell 2016), sobre todo en ecosistemas caracterizados por la presencia de periodos secos en el verano. Es decir, aunque exista un incremento en la precipitación, la evapotranspiración también sufrirá un aumento (Cook, Smerdon, Seager y Coats, 2014). Esto coincide con los incendios presentados en la Sierra Madre Occidental y Oriental, las cuales poseen un clima de tipo seco desértico y seco estepario en gran parte de su extensión (NavarCháidez y González-Elizondo, 2009). Sin embargo, las zonas que presentan mayor ocurrencia y superficie afectada por incendios forestales (i.e. Sierra Madre del Sur y Eje Neovolcánico) tienen un clima tropical con lluvias todo el verano y templado con lluvias todo el año respectivamente. Esta disponibilidad hídrica evidencia que la sequía no es el promotor de los incendios en estas regiones y puede atribuirse a otros factores (Zúñiga-Vásquez et al., 2017b).

Estudios en el norte y centro de México reportan que los incendios forestales se comportan de manera estacional (Cerano-Paredes, Villanueva-Díaz y Fulé, 2010; CeranoParedes et al., 2015). Por ejemplo, en el Norte de México las temperaturas máximas y la precipitación mínima se presentan durante la primavera (Instituto Mexicano de Tecnología del Agua [IMTA], 2009). Esta estacionalidad se ve reflejada en la ocurrencia de incendios forestales (Littell et al., 2016) y resalta la importancia del uso de datos de sequía acumulada. Cabe mencionar que los resultados mostrados en la figura 3 refuerzan la hipótesis planteada de este estudio. No obstante, estos hallazgos se deben tomar con prudencia debido a que varían espaciotemporalmente (Pompa-García et al., 2017). Una limitante en nuestro estudio es que no se consideró que la frecuencia del fuego está influenciada por las condiciones climáticas de los años previos. Las condiciones de humedad contribuyen a la producción de biomasa, lo que constituye combustible para exacerbar la conducta del fuego (Pompa-García et al., 2017).

Los resultados en este análisis se consideran útiles a la contribución de los recientes estudios sobre la influencia de la sequía en la ocurrencia de incendios forestales y la superficie afectada por ellos (Zúñiga-Vásquez et al., 2017b; Pompa-García et al., 2017); sin embargo, es necesario tomarlos con mesura debido a su posible sobrestimación (Zhu, Kobayashi, Kanaya y Saito et al., 2017) y a la cantidad de datos Modis disponibles. En relación con las áreas incendiadas Di Bella (2008) señala que la capacidad de los sensores en la detección de incendios se ve influenciada porque estos tienen menor tamaño que la unidad de observación mínima y por esta razón identifica al pixel en su totalidad como un área quemada. Algunos estudios postincendio de áreas quemadas han demostrado también que existe una sobrestimación de la superficie quemada debido a la baja resolución de Modis (Chuvieco et al., 2008). No obstante, esta sobreestimación puede verse compensada cuando no se reconocen superficies incendiadas de un tamaño menor al de la resolución del este sensor (Isabel et al., 2013). Por lo anterior se sugiere que en estudios posteriores se tomen en cuenta las limitantes descritas en este estudio para generar una mayor investigación al respecto.

\section{CONCLUSIONES}

Los periodos de sequía mostraron una relación importante con la ocurrencia e intensidad de los incendios forestales, particularmente para el caso de las superficies incendiadas; sin embargo, es conveniente realizar otros análisis complementarios como el cambio de uso del suelo, las condiciones fisiográficas del sitio y la variabilidad interanual climática. 
Los resultados presentados constituyen una alternativa para la disposición de información del fuego, dadas las estadísticas limitantes desde una perspectiva geoespacial. Los mapas de gradientes obtenidos muestran el comportamiento del fuego en las zonas fisiográficas lo que guarda consistencia con resultados encontrados en estudios recientes.

\section{RECONOCIMIENTOS}

Se agradece el apoyo de la Maestría Institucional en Ciencias Agropecuarias y Forestales (Micaf) y al Consejo Nacional de Ciencia y Tecnología (Conacyt) por el apoyo para los estudios de maestría del primer y segundo autor. Agradecemos al equipo editorial y dos árbitros anónimos cuyas aportaciones mejoraron considerablemente el manuscrito.

\section{REFERENCIAS}

Beguería, S., Latorre, B., Reig, F., \& Vicente-Serrano, S.M. (2017). SPEI Global Drought Monitor. Recuperado de http://spei.csic.es/map/maps.html\#months=1\#month= 7\#year $=2017$

Bodí, M., Cerdà, A., Mataix-Solera J., \& Doerr, S. H. (2012). Efectos de los incendios forestales en la vegetación y el suelo en la cuenca mediterránea: revisión bibliográfica. Boletín de la Asociación de Geógrafos Españoles, 58, 33-55.

Burel, F., \& Baudry, J. (2002). Ecología del paisaje: conceptos, métodos y aplicaciones. Madrid: Mundi-Prensa.

Carreiras, M., Ferreira, A. J. D., Valente, S., Fleskens, L., Gonzales-Pelayo, Ó., Rubio, J. L., Stoof, C. R., Coelho, C. O. A., Ferreira, C. S. S., \& Ritsema, C. J. (2014). Comparative analysis of policies to deal with the wildfire risk. Land Degradation \& Development, 25, 92-103. doi: 10.1002/ldr.2274

Castillo-Castillo, M., Ibáñez-Castillo, L. A., Valdés, J. B., ArteagaRamírez, R., \& Vázquez-Peña, M. A. (2017). Análisis de sequías meteorológicas en la cuenca del río Fuerte, México. Tecnología y Ciencias del Agua, 8(1), 35-52. doi: 10.24850/jtyca-2017-01-03.

Cerano-Paredes, J., Villanueva-Díaz, J., \& Fulé, P. Z. (2010). Reconstrucción de incendios y su relación con el clima para la Reserva Cerro El Mohinora, Chihuahua. Revista Mexicana de Ciencias Forestales, 1(1), 63-74.
Cerano-Paredes, J., Villanueva-Díaz, J., Cervantes-Martínez, R., Fulé, P. Z., Yocom, L., Esquivel-Arriaga, G., \& JardelPeláez, E. (2015). Historia de incendios en un bosque de pino de la sierra de Manantlán, Jalisco, México. Bosque, 36(1), 41-52. doi: 10.4067/S0717-92002015000100005

Chuvieco, E., Opazo, S., Sione, W., Valle, H. D., Anaya, J., Bella, C. D., \& González-Alonso, F. (2008). Global burned-land estimation in Latin America using MODIS composite data. Ecological Applications, 18(1), 64-79. doi: 10.1890/06-2148.1

Comisión Nacional Forestal [Conafor]. 2017. Reporte semanal de resultados de incendios forestales 2017. Programa Nacional de Prevención de Incendios Forestales. Recuperado de: https://www.gob.mx/cms/uploads/attachment/file/256 620/Reporte_del_01_de_enero_al_14_de_septiembre_de _2017.pdf.

Comisión Nacional para el Conocimiento y Uso de la Biodiversidad [Conabio]. 2017. La diversidad biológica forestal en México. Convenio sobre la Diversidad Biológica. Recuperado de: http://www.conabio.gob.mx/institucion/cooperacion_in ternacional/doctos/dbf_mexico.html.

Cook, B. I., Smerdon, J. E., Seager, R., \& Coats, S. (2014). Global warming and 21 st century drying. Climate Dynamics, 43(10), 2607-2627. https://doi.org/10.1007/s00382-014-2075-y

Del Campo P-L, A., \& Bernal del T, F. H. (2010). Incendios de cobertura vegetal y biodiversidad: una mirada a los impactos y efectos ecológicos potenciales sobre la diversidad vegetal. El hombre y la máquina, 35, 67-81.

Di Bella, C. M., Posse, G., Beget, M. E., Fischer, M. A., Mari, N., \& Veron, S. (2008). La teledetección como herramienta para la prevención, seguimiento y evaluación de incendios e inundaciones. Ecosistemas, 17(3), 39-52.

Espíndola, M. Á. C., Trejo, D. A. R., Morales, A. V., \& Pérez, J. S. (2017). Factores sociales de uso del suelo y vegetación asociados a los incendios forestales en Hidalgo. Revista Mexicana de Ciencias Forestales, 8(41), 139-163. https://doi.org/10.29298/rmcf.v8i41.29

Estrada, C., \& Ángeles, C. D. R. (2007). Evaluación de combustibles forestales en el Parque Nacional "El Chico", Hidalgo. Ecología y biodiversidad, claves de la prevención. Pachuca, Hidalgo, México. 17 p.

Flores, J., Rodríguez, D., Estrada, O., \& Sánchez, F. (2006). Incendios Forestales: Definiendo el Problema, Ecología y Manejo, Participación Social, Fortalecimiento de 
Capacidades, Educación y Divulgación. Madrid: Ediciones Mundi-Prensa

Gómez-Pazo, A., \& Salas, J. (2017). Modelado del peligro de ignición de incendios forestales en Galicia. Rev. Geo. Sur, 7(10), 1-14.

Instituto Mexicano de Tecnología del Agua [IMTA]. (2009). Extractor Rápido de Información Climatológica III (ERIC), Software. Jiutepec, Morelos, México.

Instituto Nacional de Geografía e Informática [Inegi]. (2013). Recursos Naturales. Fisiografía. Recuperado de: http://www.inegi.org.mx/geo/contenidos/recnat/usosue lo.

Instituto Nacional de Geografía e Informática [Inegi]. (2017). Recursos Naturales. Fisiografía. Recuperado de: http://www.inegi.org.mx/geo/contenidos/recnat/fisiogr afia/infoescala.aspx.

Isabel, M. M., Salinero, E. C., Pavón, P. O., Verdú, F. R., Solana, H. N., \& Paredes, D. P. (2013). A practical example of remote sensing application in the management of natural hazards: cartography and urgent evaluation of burned areas in Galicia. Cuadernos de Investigación Geográfica, 33,19-38. doi: 10.18172/cig.1187.

Jiménez, J., \& Alanís, E. (2011). Análisis de la frecuencia de los incendios forestales en la Sierra Madre Oriental y Occidental del norte de México y sur de Estados Unidos de América. Ciencia UANL, 14(3), 255-263.

Keeley, J. E. (2009). Fire intensity, fire severity and burn severity: a brief review and suggested usage. International Journal of Wildland Fire, 18(1),116. doi: 10.1071/WF07049.

Littell, J. S., Peterson, D. L., Riley, K. L., Liu, Y., \& Luce, C. H. (2016). A review of the relationships between drought and forest fire in the United States. Global Change Biology, 22(7), 2353-2369. doi: 10.1111/gcb.13275

Márquez-Linares, M. A., Jurado E., \& López-González, C. (2005). Efecto del fuego en el establecimiento de Arctostaphylos pungens Hbk., en ecosistemas templados semihúmedos de Durango, México. Madera y Bosques, 11(2), 35-48. doi: 10.21829/myb.2005.1121255

National Aeronautics and Space Administration [NASA] (2017). Moderate Resolution Imaging Spectroradiometer. Recuperado de: https://modis.gsfc.nasa.gov/ (Acceso el 05-12-17).

National Aeronautics and Space Administration [NASA] (s/f). Active fire data. Recuperado de https://earthdata.nasa.gov/earth-observation-data/nearreal-time/ firms/active-fire-data

Návar-Cháidez, J. J., \& González-Elizondo, S. (2009). Diversidad, estructura y productividad de bosques templados de Durango, México. Polibotánica, 27, 71-87.

Ord, J. K., \& Getis, A. (1992). The analysis of spatial association by use of distance statistics. Geographical Analysis, 24(3), 189206. doi: 10.1111/j.1538-4632.1992.tb00261.x.

Páramo, G. E. (2007). Análisis, diagnóstico y elaboración del mapa de susceptibilidad a los incendios de la cobertura vegetal en Colombia (Reporte final del contrato de consultoría N. ${ }^{\circ}$ 2062372). Bogotá, Colombia: Ministerio de Ambiente, vivienda y Desarrollo Territorial (MAVDT) Fondo Financiero de Proyectos de Desarrollo (Fonade).

Peña-Fernández, E., \& Valenzuela-Palma, L. (Enero, 2008). Incremento de los incendios forestales en bosques naturales y plantaciones forestales en Chile. Memorias del segundo simposio internacional sobre políticas, planificación y economía de los programas de protección contra incendios forestales: Una visión global (pp. 595612): Departamento de Agricultura de los EE. UU., Servicio Forestal, Estación de Investigación del Pacífico Suroeste. Albany^ eCalifornia

Pérez-Cabello, F., Ibarra, P., Echeverría, M. T., \& de la Riva, J. (2010). Post-fire land degradation of Pinus sylvestris L. woodlands after 14 years. Land Degradation \& Development, 21(2), 145-160. doi:10.1002/ldr.925.

Pérez-Verdín, G., Márquez-Linares, M. A., Cortés-Ortiz, A., \& Salmerón-Macías, M. (2013). Análisis espacio-temporal de la ocurrencia de incendios forestales en Durango, México. Madera y Bosques, 19(2), 37-58.

Polychronaki, A., Gitas, I. Z., \& Minchella, A. (2014). Monitoring post-fire vegetation recovery in the Mediterranean using SPOT and ERS imagery. International Journal of Wildland Fire, 23(5), 631-642. doi: 10.1071/WF12058.

Pompa-García, M., Camarero, J. J., Rodríguez-Trejo, D. A., \& Vega-Nieva, D. J. (2017). Drought and spatiotemporal variability of forest fires across Mexico. Chinese Geographical Science, 28(1), 25-37. doi: 10.1007/s11769-017-0928-0

Pompa-García, M., \& Sensibaugh, M. (2014). Ocurrencia de incendios forestales y su teleconexión con fenómenos ENSO. Ciencia UAT, 8(2), 6-10.

Reddy, C. S., Manaswini, G., Satish, K. V., Singh, S., Jha, C. S., \& Dadhwal, V. K. (2016). Conservation priorities of forest ecosystems: Evaluation of deforestation and degradation 
hotspots using geospatial techniques. Ecological Engineering, 91, 333-342. doi: 10.1016/j.ecoleng.2016.03.007

Rodríguez-Trejo, D. A. (2001). Ecología del fuego en el ecosistema de Pinus hartwegii Lindl. Revista Chapingo. Serie Ciencias Forestales y del Ambiente, 7(2), 145-151.

Rodríguez-Trejo, D. A., \& Myers, R. L. (2010). Using oak characteristics to guide fire regime restoration in Mexican pine-oak and oak forests. Ecological Restoration, 28(3), 304323. doi: 10.3368/er.28.3.304

Roy, D., Jin, Y., Lewis, P., \& Justice, C. (2005). Prototyping a global algorithm for systematic fire affected area mapping using MODIS time series data. Remote Sensing of Environment, 97(2), 137-162. doi: 10.1016/j.rse.2005.04.007.

Seager, R., Ting, M., Davis, M., Cane, M., Naik, N., Nakamura, J., \& Stahle, D. W. (2009). Mexican drought: an observational modeling and tree ring study of variability and climate change. Atmósfera, 22(1), 1-31.

Vélez, R. (2009). Los factores causantes: las fuerzas y cambios sociales y económicos. En: Convivir con los incendios forestales: lo que nos revela la ciencia. EFI Discussion Paper, 15, 24-27.

Vicente-Serrano, S. M., Beguería, S., \& López-Moreno, J. I. (2010). A multiscalar drought index sensitive to global warming: the standardized precipitation evapotranspiration index. Journal of Climate, 23, 1696-1718. doi: 10.1175/2009JCLI2909.1.

Williams, A. P., Seager, R., Macalady, A. K., Berkelhammer, M., Crimmins, M. A., Swetnam, T. W., Trugman, A. T., Buenning, N. F., Noone, D. C., McDowell, N. G., Hryniw, N. H., Mora, C., \& Rahn, T. (2015). Correlations between components of the water balance and burned area reveal new insights for predicting forest fire area in the southwest United States. International Journal of Wildland Fire, 24(1), 1426. doi: 10.1071/WF14023.
Yocom, L. L., Fulé, P. Z., Brown, P. M., Cerano-Paredes, J., Cornejo-Oviedo, E., Cortés Montaño, C., \& Skinner, C. N. (2017). Climate drives fire synchrony but local factors control fire regime change in northern Mexico. Ecosphere, 8(3), e01709. doi: doi.org/10.1002/ecs2.1709

Zhu, C., Kobayashi, H., Kanaya Y., \& Saito, M. (2017). Sizedependent validation of MODIS MCD64A1 burned area over six vegetation types in boreal Eurasia: Large underestimation in croplands. Scientific Reports, 7(4181), 1-9. doi: 10.1038/s41598-017-03739-0

Zúñiga-Vásquez, J. M., Cisneros-González, D., Pompa-García, M., Rodríguez-Trejo, D. A., \& Pérez-Verdín, G. (2017a). Modelación espacial de incendios forestales en México: una integración de dos bases de datos. Bosque, 38(3), 563-574. doi: 10.4067/S0717-92002017000300014

Zúñiga-Vásquez, J. M., Cisneros-González, D., \& Pompa-García, M. (2017b). Drought regulates the burned forest areas in Mexico: the case of 2011, a record year. Geocarto International, 1-31. doi: 10.1080/10106049.2017.1415986

Manuscrito recibido el 8 de diciembre de 2017

Aceptado el 6 de junio de 2018

Publicado el 15 de octubre de 2018

Este documento se debe citar como:

Cisneros-González, D., Zúñiga-Vásquez, J. M., \& Pompa-García, M. (2018). Actividad del fuego en áreas forestales de México a partir de sensores remotos y su sensibilidad a la sequía. Madera y Bosques, 24(3), e2431687. doi: 10.21829/ myb.2018.2431687

Madera y Bosques por Instituto de Ecología, A.C. se distribuye bajo una Licencia Creative Commons Atribución-NoComercialCompartirlgual 4.0 Internacional. 